\title{
On Type Classification of Factors Constructed as Infinite Tensor Products
}

\author{
By \\ Osamu TaKenOUChI*
}

Let $\mathrm{H}_{\nu}(\nu=1,2, \cdots)$ be Hilbert spaces, to each of which is assigned a reference vector $e_{\nu}$ with $\left\|e_{\nu}\right\|=1$. Then an infinite tensor product (incomplete infinite direct product of von Neumann [4]) $\mathrm{H}=\prod_{\nu} \otimes$ $\left(\mathrm{H}_{\nu}, e_{\nu}\right)$ is formed. Suppose further that a von Neumann algebra $\boldsymbol{M}_{\nu}$ be given on each $\mathrm{H}_{\nu}$. Each operator $T$ in $\boldsymbol{M}_{\nu}$ is extended over all of $\mathrm{H}$, which will be denoted as $\bar{T}$. All these operators $\bar{T}$ together form a von Neumann algebra on $H$, which we write as $\overline{\boldsymbol{M}}_{\nu}$. The von Neumann algebra $\boldsymbol{M}$ on $\mathrm{H}$ generated by these $\bar{M}_{2}$ 's $(\nu=1,2, \cdots)$ is what we call as the infinite tensor product of $\boldsymbol{M}_{\nu}$.

Suppose now that each $\boldsymbol{M}_{2}$ given is a factor of type I. Then, $\mathrm{H}_{\nu}$ can be decomposed as a direct product of two Hilbert spaces $\mathrm{H}_{v_{1}}, \mathrm{H}_{v_{2}}: \mathrm{H}_{2}=\mathrm{H}_{\nu_{1}} \otimes \mathrm{H}_{v_{2}}$, and $\boldsymbol{M}_{\nu}$ is thereby identified with $\boldsymbol{B}\left(\mathrm{H}_{v_{1}}\right) \otimes \boldsymbol{I}_{v_{2}}$, where $\boldsymbol{B}\left(\mathrm{H}_{v_{1}}\right)$ is the total operator algebra on $\mathrm{H}_{\nu_{1}}$ and $\mathbb{I}_{v_{2}}$ is a von Neumann algebra consisting of all scalar multiples of the identity operator on $\mathrm{H}_{22}$. With respect to this direct product decomposition, $e_{\nu}$ can be expressed as

$$
e_{\nu}=\sum_{j=1}^{m \nu} \lambda_{\nu j}{ }^{1 / 2} \psi_{\nu 1 j} \otimes \psi_{\nu 2 j}
$$

where

$$
\begin{aligned}
& \lambda_{\nu j}>0 \text { for all } j, \text { and } \lambda_{\nu 1} \geqq \lambda_{\nu 2} \geqq \cdots, \sum_{j=1}^{n_{\nu}} \lambda_{\nu j}=1 ; \\
& \psi_{\nu 1 j} \text { 's form a normalized orthogonal system in } \mathrm{H}_{\nu 1} ; \\
& \psi_{\nu 2 j} \text { 's form a normalized orthogonal system in } \mathrm{H}_{\nu 2} \text {. }
\end{aligned}
$$

Received July 12, 1968.

Communicated by H. Araki.

* Faculty of Engineering Sciences, Osaka University. 
The expression may exist in many ways, but the set of $\lambda_{\nu j}$ 's including the multiplicity of each $\lambda_{\nu j}$ remains fixed in all these expressions. So we may ask whether the type of the von Neumann algebra $\boldsymbol{M}$, which is in this case a factor, can be determined explicitly in terms of these $\lambda_{\nu j}$ 's. The following theorem constitutes an answer to this problem.

Theorem. As to the type of factor of $M$,

Type I occurs if and only if

$$
\sum_{\nu=1}^{\infty}\left(1-\lambda_{\nu_{1}}\right)<\infty
$$

Type $\mathrm{II}_{1}$ occurs if and only if $n_{\nu}$ 's are all finite and $n_{\nu}>1$ for infinitely many $n_{\nu}$, and

$$
\sum_{\nu=1}^{\infty} \sum_{j=1}^{n \nu}\left(\left(\frac{1}{n_{\nu}}\right)^{1 / 2}-\lambda_{\nu j}{ }^{1 / 2}\right)^{2}<\infty,
$$

where $n_{\nu}=\operatorname{dim} \mathrm{H}_{\nu_{1}}$, and $\lambda_{\nu j}$ 's are considered to be 0 for $j>m_{\nu}$;

Type III occurs if and only if

$$
\sum_{\nu=1}^{\infty} \sum_{j, k=1}^{m \nu} \lambda_{\cdot, j} \lambda_{\nu_{k}} \min \left\{\left|\frac{\lambda_{\nu, j}}{\lambda_{\nu_{k}}}-1\right|^{2}, c\right\}=\infty
$$

for some, and hence for all $c>0$.

Type I condition is first given by Araki [1], and Bures [2]. A simple proof is given in Moore [3]. Type $\mathrm{II}_{1}$ condition is given partly by Bures [2], and in a slightly restricted form by Moore [3]. (See below the end of Section 1.) Type III condition was only treated under a subsidiary condition that the $\lambda_{\nu_{1}}$ 's are bounded below by a positive constant. (Bures [2], Moore [3]) So our contribution is that we have removed this restriction. Note that, under the subsidiary condition mentioned above, our condition is reduced to that of Moore [3].

Our result is thus a refinement of that of Moore, and we follow his method to a great extent in the proof of "if" part. The proof of "only if" part is independent of his.

For the simplicity of writing, $\sum_{\nu}, \prod_{\nu}$ denote the summation and 
product over $\nu=1,2, \cdots . \sum_{j}, \sum_{k}$ mean the summations over $j, k=1$, $\cdots, m_{\nu}$ when $m_{\nu}<\infty$, and over $j, k=1,2, \cdots$ when $m_{\nu}=\infty$. In some places, we use $\mathrm{I}(\nu)$ to mean the set $\left\{1, \cdots, m_{\nu}\right\}$ for $m_{\nu}<\infty$ and $\{1,2, \cdots\}$ for $m_{\nu}=\infty$.

1. First we prepare some properties which will be needed.

Lemma 1. Let $\mathrm{H}_{\nu_{1}}^{0}$ be the closed subspace of $\mathrm{H}_{\nu_{1}}$ spanned by $\left\{\psi_{\nu 1 j} ; j \in \mathrm{I}(\nu)\right\}, E_{\nu 1}$ be the projection operator in $\mathrm{H}_{\nu}$ on the closed subspace $\mathrm{H}_{\nu}{ }^{0}=\mathrm{H}_{v_{1}}^{0} \otimes \mathrm{H}_{v_{2}} \cdot E_{\nu_{1}} \in \boldsymbol{M}_{\nu}$.

Let the extention of $E_{\nu_{1}}$ to $\mathrm{H}$ be $\bar{E}_{\nu 1}$, and $E=\prod_{\nu} \bar{E}_{\nu 1} . \quad E \in \boldsymbol{M}$, $E \mathrm{H}=\Pi_{\nu} \otimes\left(\mathrm{H}_{\nu}{ }^{0}, e_{\nu}\right)$, and $(\boldsymbol{M})_{E}$, the part of $\boldsymbol{M}$ in $E \mathrm{H}$, is the infinite tensor product of $(\boldsymbol{M})_{E_{v_{1}}}$.

Suppose that the type of factor of $(\boldsymbol{M})_{E}$ is already settled. (Observe that the determination of the type of factor of $(\boldsymbol{M})_{E}$ is rather easy due to the fact that the length of the expression (1) and $\operatorname{dim} \mathrm{H}_{v 1}^{0}$ are equal, or, in other words, $\left\{\psi_{v_{1 j}} ; j \in I(\nu)\right\}$ is a complete normalized orthogonal system in $\mathrm{H}_{v_{1}}^{0}$, and this fact makes strict the relation between the infinite tensor product and the measure theoretic construction. (See the first few passages in Section 2.))

According to that the type of $(\boldsymbol{M})_{E}$ is I, II, or III, we know the type of $\boldsymbol{M}$ is I, II, or III.

When $(\boldsymbol{M})_{E}$ is a finite factor, $\boldsymbol{M}$ is a finite factor if and only if all $n$ 's are finite, and

$$
\sum_{\nu}\left(1-\frac{m_{\nu}}{n_{\nu}}\right)<\infty
$$

Proof. The last assertion only is to be proved.

It is clear that, if one of $n_{\nu}$ 's is $\infty, \boldsymbol{M}$ is an infinite factor. So we suppose that all the $n_{\nu}$ 's are finite, and $\sum_{\nu}\left(1-\frac{m_{\nu}}{n_{\nu}}\right)=\infty$. This implies that $\prod_{\nu} \frac{n_{\nu}}{m_{\nu}}=\infty$. Therefore, there exist disjoint finite subsets $A_{k}$ of positive integers such that $\prod_{\nu \in A_{k}} \frac{n_{\nu}}{m_{\nu}} \geqq 2$ for each $k(=1,2, \cdots)$. Then, $\prod_{\nu \in A_{k}} \otimes \mathrm{H}_{\nu 1}$ contains a projection $F_{k 1}$ such that $F_{k}=F_{k 1} \otimes I_{k 2} \in \prod_{\nu \in A_{k}}$ $\otimes \boldsymbol{M}_{\nu}$, dim range $\left(F_{k 1}\right)=\prod_{\nu \in A_{k}} m_{\nu}, F_{k_{\nu \in A_{k}}} \otimes E_{\nu 1}=0$. Consider projections 
$\bar{G}_{k}(k=1,2, \cdots)$ in $\mathrm{H}$ defined by $\bar{G}_{k}=\bar{F}_{k_{\nu \notin A_{k}}} \bar{E}_{\nu 1}$. Then, $E, \bar{G}_{1}, \bar{G}_{2}, \cdots$ are mutually orthogonal projections in $\boldsymbol{M}$ which are mutually equivalent with respect to $\boldsymbol{M}$. So $\boldsymbol{M}$ cannot be finite.

Suppose now $\sum_{\nu=1}^{\infty}\left(1-\frac{m_{\nu}}{n_{\nu}}\right)<\infty . \quad \boldsymbol{M}$ is in any way semi-finite, and we consider a dimension funcsion d on $\boldsymbol{M}$. As $(\boldsymbol{M})_{E}$ is finite, $E$ is a finite projection, so that $0<\mathrm{d}(E)<\infty$. Normalize $\mathrm{d}$ in such a way that $\mathrm{d}(E)=\Pi_{\nu} \frac{m_{\nu}}{n_{\nu}}$. Now by the construction of infinite tensor product $\Pi_{\nu} \otimes\left(H_{\nu}, e_{v}\right)$, we have $I=\sum_{\left(\varepsilon_{\nu}\right)} E_{\left(\varepsilon_{\nu}\right)}$, where $\left(\varepsilon_{\nu}\right)=\left(\varepsilon_{1}, \varepsilon_{2}, \cdots\right)$ is a sequence consisting of 0 's and a finite number of 1 's, and $E_{\left(\varepsilon_{\nu}\right)}=\prod_{\nu} \otimes \bar{E}_{\nu 1 \varepsilon_{\nu}}$ with $E_{v 10}=E_{\imath 1}, E_{v 11}=I_{\nu 1}-E_{\gamma_{1}}$. These $E_{\left(\varepsilon_{\nu}\right)}$ 's are all finite projections with $\mathrm{d}\left(E_{\left(\varepsilon_{\nu}\right)}\right)=\mathrm{d}(E) \prod_{\nu}\left(\frac{n_{\nu}-m_{\nu}}{m_{\nu}}\right)^{\varepsilon_{\nu}}=\left(\prod_{\nu: \varepsilon_{\nu}=0} \frac{m_{\nu}}{n_{\nu}}\right)\left(\prod_{\nu: \varepsilon_{\nu}-1}\left(1-\frac{m_{\nu}}{n_{\nu}}\right)\right)$. Thus, summed up all together, $\mathrm{d}(I)=1$, and $\boldsymbol{M}$ is finite.

Lemma 2. For each $\nu(=1,2, \cdots)$, consider a subset $\mathrm{I}^{\prime}(\nu)$ of $\mathrm{I}(\nu)$ such that

$$
\sum_{\nu} \sum_{j \in \mathrm{I}^{\prime}(\nu)} \lambda_{v j}<\omega .
$$

The $\nu$ 's such as $\mathrm{I}^{\prime}(\nu)=\mathrm{I}(\nu)$ amount only finite, and we may leave them out of consideration. So, let

$$
\mathrm{J}(\nu)=\mathrm{I}(\nu)-\mathrm{I}^{\prime}(\nu) \neq \phi .
$$

Define for $j \in \mathrm{J}(\nu)$

Then

$$
\begin{aligned}
& \lambda_{\nu_{j}}^{\prime}=\lambda_{\nu_{j}} / \sum_{k \in \mathrm{J}(\nu)} \lambda_{\nu_{k}}, \\
& e_{\nu}^{\prime}=\sum_{j \in \mathrm{J}(\nu)} \lambda_{\nu_{j}}^{\prime 1 / 2} \psi_{v_{1 j}} \otimes \psi_{\nu 2 j} .
\end{aligned}
$$

$$
\mathrm{H}=\prod_{\nu} \otimes\left(\mathrm{H}_{\nu}, e_{\nu}\right)=\prod_{\nu} \otimes\left(\mathrm{H}_{\nu}, e_{\nu}{ }^{\prime}\right) .
$$

Therefore, $\boldsymbol{M}$ is also not affected by this change of reference vectors, and moreover, convergence conditions (5), (6), (7) are affected neither.

Proof. The condition that two sets of reference vectors $\left\{e_{\nu} ; \nu=\right.$ $1,2, \cdots\}$ and $\left\{e_{\nu}{ }^{\prime} ; \nu=1,2, \cdots\right\}$ determine the same infinite tensor product is the convergence of $\sum_{\nu}\left(1-\left(e_{\nu}, e_{\nu}{ }^{\prime}\right)\right.$ ). (von Neumann [4]) 
In our case, this certainly holds as

$$
\begin{aligned}
\sum_{\nu}\left(1-\left(e_{\nu}, e^{\prime}{ }_{\nu}\right)\right) & =\sum_{\nu}\left(1-\sum_{j \in \mathrm{J}(\nu)} \lambda_{\nu j}{ }^{1 / 2} \lambda_{\nu j}{ }^{1 / 2}\right) \\
& =\sum_{\nu}\left(1-\left(\sum_{j \in \mathrm{J}(\nu)} \lambda_{\nu j}\right)^{1 / 2}\right) \\
& \leqq \sum_{\nu}\left(1-\sum_{j \in \mathrm{J}(\nu)} \lambda_{\nu j}\right)=\sum_{\nu} \sum_{j \in \mathrm{I}^{\prime}(\nu)} \lambda_{\nu j}<\infty .
\end{aligned}
$$

For the proof of the latter part, we remark first that, from (8),

$$
\sum_{j \in \mathrm{J}(\nu)} \lambda_{\nu_{j}} \geqq \frac{1}{2} \quad \text { for large } \nu \text {. }
$$

So, we restrict ourselves to those $\nu$ 's which satisfy (9).

Ad (5). In this case, $\lambda_{\nu_{1}}^{\prime}$ is to be considered as the largest member among $\lambda_{\nu j}^{\prime}(j \in \mathrm{J}(\nu))$.

When $\sum_{\nu}\left(1-\lambda_{\nu_{1}}\right)<\infty, \lim _{\nu \rightarrow \infty} \lambda_{\nu_{1}}=1$. And by $(9), 1 \in J(\nu)$ for large $\nu$. For such $\nu, \lambda_{\nu_{1}}^{\prime}=\lambda_{\nu_{1}} / \sum_{j \in \mathrm{J}(\nu)} \lambda_{\nu_{j}} \geqq \lambda_{\nu_{1}}$, and $\sum_{\nu}\left(1-\lambda_{\nu_{1}}^{\prime}\right)<\infty$.

Suppose now that $\sum_{\nu}\left(1-\lambda_{\nu_{1}}^{\prime}\right)<\infty$. Let $j_{\nu}$ be the smallest integer in $\mathrm{J}(\nu)$. Then, $\lambda_{\nu_{1}}^{\prime}=\lambda_{\nu_{j}} / \sum_{k \in \mathrm{J}(\nu)} \lambda_{\nu k}$.

As

$$
\begin{aligned}
& 1-\lambda_{\nu_{1}}^{\prime}+\sum_{k \in I^{\prime}(\nu)} \lambda_{\nu_{k}}+2\left(\sum_{k \in I^{\prime}(\nu)} \lambda_{\nu_{k}}\right)^{2} \\
= & 1-\frac{\lambda_{\nu_{\nu}}}{\sum_{k \in J(\nu)} \lambda_{\nu k}}+\sum_{k \in \mathrm{I}^{\prime}(\nu)} \lambda_{\nu_{k}}+2\left(\sum_{k \in \mathrm{I}^{\prime}(\nu)} \lambda_{\nu k}\right)^{2} \\
= & \left(\sum_{k \in \mathrm{J}(\nu)-\left\{j_{\nu}\right\}} \lambda_{\nu k}+\sum_{k \in \mathrm{I}^{\prime}(\nu)} \lambda_{\nu_{k}}-\left(\sum_{k \in I^{\prime}(\nu)} \lambda_{\nu_{k}}\right)^{2}\right) / \sum_{k \in \mathrm{J}(\nu)} \lambda_{\nu_{k}}+2\left(\sum_{k \in \mathrm{I}^{\prime}(\nu)} \lambda_{\nu_{k}}\right)^{2} \\
\geqq & 1-\lambda_{\nu_{j}} \geqq 1-\lambda_{\nu_{1}},
\end{aligned}
$$

$\sum_{\nu}\left(1-\lambda_{\nu_{1}}\right)<\infty$, the condition (8) being taken into consideration.

Ad (6). $\quad n_{\nu}$ 's must be considered as finite for all $\nu(=1,2, \cdots)$.

Now, setting $\lambda_{\nu j}^{\prime}=0$ for $j \notin \mathrm{J}(\nu)$,

$$
\begin{aligned}
& \left|\left(\sum_{\nu} \sum_{j=1}^{n_{\nu}}\left(\left(\frac{1}{n_{\nu}}\right)^{1 / 2}-\lambda_{\nu j}{ }^{1 / 2}\right)^{2}\right)^{1 / 2}-\left(\sum_{\nu} \sum_{j=1}^{n \nu}\left(\left(\frac{1}{n_{\nu}}\right)^{1 / 2}-\lambda_{\nu j}{ }^{1 / 2}\right)^{2}\right)^{1 / 2}\right| \\
\leqq & \left(\sum_{\nu} \sum_{j \in \mathrm{I}(\nu)}\left(\lambda_{\nu j}^{\prime}{ }^{1 / 2}-\lambda_{\nu j}{ }^{1 / 2}\right)^{2}\right)^{1 / 2} \\
\leqq & \left(\sum_{\nu} \sum_{j \in \mathrm{J}(\nu)}\left(\frac{\lambda_{\nu j}{ }^{1 / 2}}{\left(\sum_{k \in \mathrm{J}(\nu)} \lambda_{\nu k}\right)^{1 / 2}}-\lambda_{\nu j}{ }^{1 / 2}\right)^{2}\right)^{1 / 2}+\left(\sum_{\nu} \sum_{j \in \mathrm{I}^{\prime}(\nu)} \lambda_{\nu j}\right)^{1 / 2}
\end{aligned}
$$




$$
\begin{aligned}
& =\left(\sum_{\nu} \sum_{j \in \mathrm{J}(\nu)} \frac{\lambda_{\nu j}}{\sum_{k \in \mathrm{J}(\nu)} \lambda_{\nu k}}\left(1-\left(\sum_{k \in \mathrm{J}(\nu)} \lambda_{\nu k}\right)^{1 / 2}\right)^{2}\right)^{1 / 2}+\left(\sum_{\nu} \sum_{j \in I^{\prime}(\nu)} \lambda_{\nu j}\right)^{1 / 2} \\
& =\left(\sum_{\nu}\left(1-\left(\sum_{k \in \mathrm{J}(\nu)} \lambda_{\nu k}\right)^{1 / 2}\right)^{2}\right)^{1 / 2}+\left(\sum_{\nu} \sum_{j \in I^{\prime}(\nu)} \lambda_{\nu j}\right)^{1 / 2} \\
& =\left(\sum_{\nu}\left(1+\sum_{k \in \mathrm{J}(\nu)} \lambda_{\nu k}-2\left(\sum_{k \in \mathrm{J}(\nu)} \lambda_{\nu k}\right)^{1 / 2}\right)\right)^{1 / 2}+\left(\sum_{\nu} \sum_{j \in I^{\prime}(\nu)} \lambda_{\nu j}\right)^{1 / 2} \\
& \leqq\left(2 \sum_{\nu}\left(1-\left(\sum_{k \in \mathrm{J}(\nu)} \lambda_{\nu k}\right)^{1 / 2}\right)\right)^{1 / 2}+\left(\sum_{\nu} \sum_{j \in I^{\prime}(\nu)} \lambda_{\nu j}\right)^{1 / 2} .
\end{aligned}
$$

And, because $\sum_{\nu} \sum_{k \in I^{\prime}(\nu)} \lambda_{\nu k}=\sum_{\nu}\left(1-\sum_{k \in \mathrm{J}(\nu)} \lambda_{\nu_{k}}\right)$ and $\sum_{\nu}\left(1-\left(\sum_{k \in \mathrm{J}(\nu)} \lambda_{\nu k}\right)^{1 / 2}\right)$ converge at the same time, the last member in the above sequence of expressions is finite. Therefore, if one of $\sum_{\nu} \sum_{j=1}^{n \nu}\left(\left(\frac{1}{n_{\nu}}\right)^{1 / 2}-\lambda_{\nu_{j}}{ }^{1 / 2}\right)^{2}$ and $\sum_{\nu} \sum_{j=1}^{n \nu}\left(\left(\frac{1}{n_{\nu}}\right)^{1 / 2}-\lambda_{\nu j}^{\prime 1 / 2}\right)^{2}$ is convergent, so is the other.

Ad (7). Under (9), we have

$$
\lambda_{\nu_{j}} \lambda_{v_{k}} \leqq \lambda_{\nu_{j}}^{\prime} \lambda_{\nu_{k}}^{\prime} \leqq 4 \lambda_{\nu_{j}} \lambda_{\nu_{k}} \quad \text { for } \quad j, k \in \mathrm{J}(\nu)
$$

Therefore,

And,

$$
\begin{aligned}
& \sum_{\nu} \sum_{j, k} \lambda_{\nu j} \lambda_{\nu k} \min \left\{\left|\frac{\lambda_{\nu j}}{\lambda_{\nu k}}-1\right|^{2}, c\right\} \\
& =\sum_{\nu}\left(\sum_{j, k \in J(\nu)} \lambda_{\nu_{j}} \lambda_{\nu k} \min \left\{\left|\frac{\lambda_{\nu j}}{\lambda_{\nu k}}-1\right|^{2}, c\right\}\right. \\
& +\sum_{j \in \mathrm{I}^{\prime}(\nu)} \lambda_{\nu_{j}} \sum_{k \in \mathrm{J}(\nu)} \lambda_{\nu_{k}} \min \left\{\left|\frac{\lambda_{\nu j}}{\lambda_{\nu_{k}}}-1\right|^{2}, c\right\} \\
& +\sum_{k \in \mathrm{I}^{\prime}(\nu)} \lambda_{\nu k} \sum_{j \in \mathrm{J}(\nu)} \lambda_{\nu_{j}} \min \left\{\left|\frac{\lambda_{\nu j}}{\lambda_{\nu_{k}}}-1\right|^{2}, c\right\} \\
& \left.+\sum_{j, k \in I^{\prime}(\nu)} \lambda_{\nu j} \lambda_{\nu k} \min \left\{\left|\frac{\lambda_{\nu j}}{\lambda_{\nu_{k}}}-1\right|^{2}, c\right\}\right) \\
& \leqq \sum_{\nu} \sum_{j, k \in \mathrm{J}(\nu)} \lambda_{\nu_{j}}^{\prime} \lambda_{\nu_{k}}^{\prime} \min \left\{\left|\frac{\lambda_{\nu_{j}}^{\prime}}{\lambda_{\nu_{k}}^{\prime}}-1\right|^{2}, c\right\} \\
& +2 c \sum_{\nu} \sum_{j \in I^{\prime}(\nu)} \lambda_{\cdot, j}+c \sum_{\nu}\left(\sum_{j \in I^{\prime}(\nu)} \lambda_{, j}\right)^{2} .
\end{aligned}
$$

$$
\begin{aligned}
& \sum_{\nu} \sum_{j, \mathrm{I}^{\prime}(\nu)} \lambda_{\nu_{j}}^{\prime} \lambda_{\nu_{k}}^{\prime} \min \left\{\left|\frac{\lambda_{\nu, j}^{\prime}}{\lambda_{\nu_{k}}^{\prime}}-1\right|^{2}, c\right\} \\
& \leqq 4 \sum_{\nu} \sum_{j, k \in \mathrm{I}(\nu)} \lambda_{\nu j} \lambda_{\nu k} \min \left\{\left|\frac{\lambda_{\nu j}}{\lambda_{\nu k}}-1\right|^{2}, c\right\} \text {. }
\end{aligned}
$$

Thus, by taking (8) into account, the condition on $\lambda_{\nu_{j}}$ and that of $\lambda_{\nu_{j}}^{\prime}$ are equivalent. 
Here we add a comment concerning the type $\mathrm{II}_{1}$ condition of our Theorem. Indeed, a direct application of Moore's result and Lemma 1 in the above will establish that $\boldsymbol{M}$ is of type $\mathrm{II}_{1}$ if and only if infinitely many $m_{\nu}$ 's are $>1$ and following two conditions are satisfied:

$$
\begin{aligned}
\sum_{\nu} \sum_{j=1}^{m_{\nu}}\left(\left(\frac{1}{m_{\nu}}\right)^{1 / 2}-\lambda_{\nu j}{ }^{1 / 2}\right)^{2} & <\infty, \\
\sum_{\nu}\left(1-\frac{m_{\nu}}{n_{\nu}}\right) & <\infty .
\end{aligned}
$$

As we have,

$$
\begin{aligned}
& \left(\left(\sum_{\nu} \sum_{j=1}^{n_{\nu}}\left(\left(\frac{1}{n_{\nu}}\right)^{1 / 2}-\lambda_{\nu j}{ }^{1 / 2}\right)^{2}\right)^{1 / 2}-\left(\sum_{\nu} \sum_{\nu=1}^{m_{\nu}}\left(\left(\frac{1}{m_{\nu}}\right)^{1 / 2}-\lambda_{\nu j}{ }^{1 / 2}\right)^{2}\right)^{1 / 2}\right)^{2} \\
\leqq & \sum_{\nu} \sum_{\nu}\left(\left(\frac{1}{n_{\nu}}\right)^{1 / 2}-\left(\frac{1}{m_{\nu}}\right)^{1 / 2}\right)^{2}+\sum_{\nu} \frac{n_{\nu}-m_{\nu}}{n_{\nu}} \\
= & \sum_{\nu} m_{\nu}\left(\left(\frac{1}{n_{\nu}}\right)^{1 / 2}-\left(\frac{1}{m_{\nu}}\right)^{1 / 2}\right)^{2}+\sum_{\nu}\left(1-\frac{m_{\nu}}{n_{\nu}}\right) \\
= & \sum_{\nu}\left(1+\frac{m_{\nu}}{n_{\nu}}-2\left(\frac{m_{\nu}}{n_{\nu}}\right)^{1 / 2}\right)+\sum_{\nu}\left(1-\frac{m_{\nu}}{n_{\nu}}\right) \\
\leqq & 2 \sum_{\nu}\left(1-\left(\frac{m_{\nu}}{n_{\nu}}\right)^{1 / 2}\right)+\sum_{\nu}\left(1-\frac{m_{\nu}}{n_{\nu}}\right) \\
\leqq & 3 \sum_{\nu}\left(1-\frac{m_{\nu}}{n_{\nu}}\right)
\end{aligned}
$$

conditions (6) and (10) are equivalent when (11) holds.

And (11) will surely hold when (6) is valid, because

$$
\begin{aligned}
\infty & >\sum_{\nu} \sum_{j=1}^{n_{\nu}}\left|\left(\frac{1}{n_{\nu}}\right)^{1 / 2}-\lambda_{\nu j}{ }^{1 / 2}\right|^{2} \\
& \geqq \sum_{\nu} \sum_{j=m_{\nu}+1}^{n_{\nu}} \frac{1}{n_{\nu}}=\sum_{\nu}\left(1-\frac{m_{\nu}}{n_{\nu}}\right) .
\end{aligned}
$$

In this case, infinitely many $m_{\nu}$ 's are $>1$, for we have $\lim _{\nu \rightarrow \infty} m_{\nu} / n_{\nu}=1$ from the convergence of $\sum_{\nu}\left(1-m_{\nu} / n_{\nu}\right)$ and infinitely many $n_{\nu}$ 's are supposed $>1$.

2. As Bures has stated, the type classification problem of $M$ is reduced to the following form. 
Suppose that $\Omega_{\nu}$ is a space containing $m_{\nu}$ points $\omega_{\nu_{1}}, \omega_{\nu_{2}}, \cdots, \omega_{\nu_{m_{\nu}}}$. (We here give an exposition valid when $m_{v}$ is finite. By adding a minor modification, the case where $m_{\nu}=\infty$ is also easily treated.) Consider a discrete measure $\mu_{\nu}$ on $\Omega_{\nu}$ such that $\mu_{\nu}\left(\left\{\omega_{\nu j}\right\}\right)=\lambda_{\nu_{j}}$ $\left(j=1, \cdots, m_{\nu}\right)$, and a group of transformation $G_{\nu}=\left\{g_{v_{0}}, g_{v_{1}}, \cdots, g_{v_{m_{\nu}-1}}\right\}$ such that $\omega_{v_{j}} g_{v_{k}}=\omega_{\nu(j+k)}$, where, of course, $j+k$ is considered modulo $m_{\nu}$. Let $\Omega=\prod_{\nu} \Omega_{\nu}$, and consider on $\Omega$ the product measure $\mu=\prod_{\nu} \mu_{\nu}$. Let $G$ be the restricted direct product $\prod_{\nu}^{\prime} G_{\nu}$, which acts naturally on $\Omega$ as a measurable transformation group.

Now $M$ is of type III if and only if this measure $\mu$ is not equivalent to any $G$-invariant $\sigma$-finite measure on $\Omega$ provided with a natural Borel field of subsets. Indeed, though a precise copy of his assertion will need the restriction $\operatorname{dim} \mathrm{H}_{\nu_{1}}=m_{\nu}$, it is not indispensable owing to Lemma 1 . Therefore, the "if" part is proved when one shows that, in case there exists a $G$-invariant measure $\rho$ on $\Omega$ equivalent to $\mu$, the series in (7) converges.

Let $x(\omega)=\frac{d \rho}{d \mu}(\omega)$, so that $x$ is a Borel function on $\Omega$, unique up to $\mu$ null sets and $>0$ almost everywhere. We have $\rho=\rho_{\nu} \times \rho_{\nu}{ }^{*}$, where $\rho_{\nu}$ is a measure on $\Omega_{\nu}$ giving mass 1 to each point and $\rho_{\nu}{ }^{*}$ is a measure on $\Omega_{\nu}{ }^{*}=\prod_{\iota \neq \nu} \Omega_{\iota}$, invariant under $G_{\nu}{ }^{*}=\prod_{\iota \neq \nu} G_{\iota}$. And, since $\mu=\mu_{\nu} \times \mu_{\nu}{ }^{*}, \mu_{\nu}{ }^{*}=\prod_{\iota \neq \nu} \mu_{\iota}$, we see that $\frac{d \rho}{d \mu}=\frac{d \rho_{\nu}}{d \mu_{\nu}} \times \frac{d \rho_{\nu}{ }^{*}}{d \mu_{\nu}{ }^{*}}$. To be precise, if $\omega \in \Omega, \omega=\left(\omega_{\nu} \omega_{\nu}{ }^{*}\right)$, then $x(\omega)=x_{\nu}\left(\omega_{\nu}\right) x_{\nu}{ }^{*}\left(\omega_{\nu}{ }^{*}\right)$ for almost all pairs $\left(\omega_{\nu} \omega_{\nu}{ }^{*}\right)$, where $x_{\nu}\left(\omega_{\nu}\right)=\frac{d \rho_{\nu}}{d \mu_{\nu}}\left(\omega_{\nu}\right)$ and $x_{\nu}{ }^{*}\left(\omega_{\nu}{ }^{*}\right)=\frac{d \rho_{\nu}{ }^{*}}{d \mu_{\nu}{ }^{*}}\left(\omega_{\nu}{ }^{*}\right)$. Since $\mu_{\nu}$ is discrete, this holds for almost all $x_{\nu}{ }^{*}$ for each $x_{\nu} \in \Omega_{\nu}$. We can repeat this argument to find that, for any positive integer $N$, $\rho=\prod_{\nu=1} \rho_{\nu} \times \rho_{N}^{*}$ for some measure $\rho_{N}^{*}$ on $\Omega_{N}^{*}=\prod_{\nu=N+1}^{\infty} \Omega_{\nu}$ invariant under $\prod_{\nu>N}^{\prime} G_{\nu}$. Thus, $x(\omega)=\left(\prod_{\nu=1}^{\infty} x_{\nu}\left(\omega_{\nu}\right)\right) x_{N}^{*}\left(\omega_{N}^{*}\right)$, where $\omega=\left(\omega_{1}, \cdots, \omega_{N}, \omega_{N}^{*}\right)$.

Now let $\chi^{t}, \chi_{\nu}^{t}, \chi_{N}^{* t}$ denote respectively the functions $\exp (2 \pi i t$ $\log (\cdot))$, where $(\cdot)$ is $x, x_{\nu}, x_{N}^{*}$. These functions are of absolute value 1 and

$$
\chi^{t}(\omega)=\prod_{\nu=1}^{N} \chi_{\nu}{ }_{\nu}\left(\omega_{\nu}\right) \chi_{N}^{* t}\left(\omega_{N}^{* t}\right)
$$


We view each of these functions as an element of $L^{2}(\Omega, \mu)$ by making them constant in the variables on which they do not depend. Since $\mu(\Omega)=1$, these are all unit vectors.

Now denote by $P_{N}$ the orthogonal projection of $L^{2}(\Omega)$ onto $L^{2}\left(\Omega_{N}\right)$ where $\Omega_{N}=\prod_{\nu=1}^{N} \Omega_{\nu}$ and we view $L^{2}\left(\Omega_{N}\right)$ as the subspace of $L^{2}(\Omega)$ of all functions which depend only on the coordinates $\omega_{\nu}(\nu=1, \cdots, N)$ of the point $\omega \in \Omega$. It is immediate that

$$
P_{N}\left(\chi^{t}\right)=\left(\prod_{\nu=1}^{N} \chi_{\nu}^{t}\right) \cdot\left(\chi_{N}^{* t}, 1\right),
$$

where 1 denotes the function equal to one everywhere. Then,

$$
\lim _{N \rightarrow \infty}\left\|P_{N}\left(\chi^{t}\right)-\chi^{t}\right\|=0,
$$

where $\|\cdot\|$ is the norm in $L^{2}(\Omega)$. Therefore,

$$
\left\|P_{N}\left(\chi^{t}\right)\right\|=\left|\left(\chi_{N}^{* t}, 1\right)\right| \rightarrow 1,
$$

and in particular $\left(\chi_{N}^{* t}, 1\right) \neq 0$ for large $N$. It follows at once that $\left(\chi_{\nu}{ }^{t}, 1\right)=0$ can hold for only a finite number of $\nu$. Now let us choose constants $\alpha_{v}{ }^{t}=\exp \left(2 \pi i t \beta_{v}{ }^{t}\right)$ such that

$$
\alpha_{\nu}{ }^{t}\left(\chi_{\nu}{ }^{t}, 1\right) \geqq 0 \text {. }
$$

We write then

$$
P_{N}\left(\chi^{t}\right)=\left(\prod_{\nu=1}^{N} \alpha_{\nu}{ }^{t} \chi_{\nu}{ }^{t}\right) \cdot\left(\prod_{\nu=1}^{N} \alpha_{\nu}{ }^{t}\right)^{-1}\left(\chi_{N}^{* t}, 1\right),
$$

and put

$$
\gamma_{N}^{t}=\left(\prod_{\nu=1}^{N} \alpha_{\nu}^{t}\right)^{-1}\left(\chi_{N}^{* t}, 1\right) .
$$

We observe that by our construction, for large $N$, the argument of $\gamma_{N}^{t}$ independent of $N$. If we put

$$
\delta^{t}=\bar{\gamma}_{N}^{t} /\left|\gamma_{N}^{t}\right|
$$

for large $N$, then

$$
P_{N}\left(\delta^{t} \chi^{t}\right)=\left|\gamma_{N}^{t}\right| \prod_{\nu=1}^{N} \alpha_{\nu}{ }^{t} \chi_{\nu}{ }^{t} .
$$

According to (12), we have $\lim _{N \rightarrow \infty}\left|\gamma_{N}^{t}\right|=1$, and we immediately conclude that 


$$
\prod_{\nu=1}^{N} \alpha_{\nu}{ }_{\nu} \chi_{\nu}{ }^{t} \quad \text { converges in } L^{2}(\Omega) \text {. }
$$

But (13) is equivalent to that

$$
\sum_{\nu}\left(1-\alpha_{\nu}{ }^{t}\left(\chi_{\nu}{ }^{t}, 1\right)\right) \quad \text { converges , }
$$

which in turn is equivalent to that

$$
\begin{aligned}
& \sum_{\nu}\left\|1-\alpha_{\nu}{ }^{t} \chi_{\nu}{ }^{t}\right\|^{2}<\infty, \\
& \sum_{\nu} \Im \alpha_{\nu}{ }^{t}\left(\chi_{\nu}{ }^{t}, 1\right) \quad \text { and }
\end{aligned}
$$

Thus (14) is valid for every real $t$. Now we explicit in this relation the functions $\chi_{\nu}{ }^{t}$. We have then

$$
\sum_{\nu} \sum_{j} \lambda_{\nu_{j}}\left|\exp \left(2 \pi i t\left(\beta_{\nu}{ }^{t}-\log \lambda_{\nu_{j}}\right)\right)-1\right|^{2}<\infty .
$$

Just up to this point, we followed exactly Moore. As our aim is to show the convergence of the series in (7), we consider here the following sum:

$$
\sum_{\nu_{, j, k}} \lambda_{\nu_{j}} \lambda_{\nu_{k}}\left|\exp \left(2 \pi i t\left(\log \lambda_{\nu_{j}}-\log \lambda_{\nu_{k}}\right)\right)-1\right|^{2} .
$$

As

$$
\begin{aligned}
& \left|\exp \left(2 \pi i t\left(\log \lambda_{\nu j}-\log \lambda_{\nu_{k}}\right)\right)-1\right|^{2} \\
= & \left|\exp \left(2 \pi i t\left(\beta_{\nu}{ }^{t}-\log \lambda_{v_{k}}\right)-2 \pi i t\left(\beta_{\nu}{ }^{t}-\log \lambda_{\nu_{j}}\right)\right)-1\right|^{2} \\
\leqq & 2\left(\left|\exp \left(2 \pi i t\left(\beta_{\nu}{ }^{t}-\log \lambda_{\nu_{j}}\right)\right)-1\right|^{2}\right. \\
& \left.+\left|\exp \left(2 \pi i t\left(\beta_{\nu}{ }^{t}-\log \lambda_{v_{k}}\right)\right)-1\right|^{2}\right),
\end{aligned}
$$

we immediately see that the expression (16) is dominated by

$$
\begin{aligned}
& 4 \sum_{\nu, j, k} \lambda_{\nu_{j}} \lambda_{\nu_{k}}\left|\exp \left(2 \pi i t\left(\beta_{\nu}{ }^{t}-\log \lambda_{\nu_{j}}\right)\right)-1\right|^{2} \\
= & 4 \sum_{\nu, j} \lambda_{\nu_{j}}\left|\exp \left(2 \pi i t\left(\beta_{\nu}{ }^{t}-\log \lambda_{\nu j}\right)\right)-1\right|^{2}
\end{aligned}
$$

which converges by the grace of (15).

Now the tool of which Moore made use to attain the goal can be applied here. Namely he states that the convergence of $2 \sum_{\nu} \xi_{\nu}\left(1-\cos t \eta_{\nu}\right)=\sum_{\nu} \xi_{\nu}\left|\exp i t \eta_{\nu}-1\right|^{2}$ (the $\xi_{\nu}$ 's are supposed positive) for all real $t$ is equivalent to the convergence of $\sum_{\nu} \xi_{\nu} \min \left\{\left|\eta_{\nu}\right|^{2}, c\right\}$ for a $c>0$. Applied to our case, the convergence of (16) for every 
real $t$ is equivalent to the convergence of

$$
\sum_{\nu, j, k} \lambda_{\nu_{j}} \lambda_{\nu_{k}} \min \left\{\left|\log \lambda_{\nu_{j}}-\log \lambda_{\nu_{k}}\right|^{2}, c\right\} \quad \text { for a } c>0,
$$

which in turn is equivalent to the convergence of

$$
\sum_{\nu, j, k} \lambda_{\nu_{j}} \lambda_{\nu_{k}} \min \left\{\left|\frac{\lambda_{\nu_{j}}}{\lambda_{\nu_{k}}}-1\right|^{2}, c\right\} .
$$

Thus (7) does not hold, and this completes the proof of "if" part.

3. Now, we proceed to the proof of "only if" part. To do this, we leave from the measure theoretic version of Section 2, and we treat the problem in its original form.

We assume that

$$
\begin{aligned}
\sum_{\nu, j, k} \lambda_{\nu_{j}} \lambda_{\nu k} \min \left\{\left|\frac{\lambda_{\nu \jmath}}{\lambda_{\nu k}}-1\right|^{2}, c\right\}<\infty & \\
& \text { for some, and hence for all } c>0,
\end{aligned}
$$

and we contend that $M$ is a semi-finite factor.

Assume first that there exists an $\varepsilon>0$ such that

$$
\frac{\lambda_{\nu j}}{\lambda_{\nu k}} \geqq \varepsilon \quad \text { for any } \quad \nu=1,2, \cdots, \text { and } j, k \in \mathrm{I}(\nu) \text {. }
$$

This condition being imposed, we no more need to take in (17) the minimum with some $c>0$, and so

$$
\sum_{\nu, j, k} \lambda_{\nu j} \lambda_{\nu k}\left|\frac{\lambda_{\nu j}}{\lambda_{\nu_{k}}}-1\right|^{2}<\infty
$$

Now (18) implies that $\lambda_{\nu j} \geqq \varepsilon \lambda_{\nu_{k}}$ for any $j, k=1, \cdots, m_{\nu}$. Added with respect to $j, 1 \geqq m_{\nu} \varepsilon \lambda_{\nu k}$, so $m_{\nu}$ 's are all finite. Added with respect to $k, m_{\nu} \lambda_{\nu j} \geqq \varepsilon$, so that $\lambda_{\nu j} \geqq \varepsilon / m_{\nu}$ for any $j=1, \cdots, m_{\nu}$. Using also the fact that the mean square deviation becomes the least when we take the mean value as the center, we reform the series in (19).

$$
\begin{aligned}
& \sum_{\nu, j, k} \lambda_{\nu j} \lambda_{\nu k}\left|\frac{\lambda_{\nu j}}{\lambda_{\nu k}}-1\right|^{2} \\
= & \sum_{\nu, j} \lambda_{\nu j} \sum_{k=1}^{m \nu} \lambda_{\nu k}\left|\frac{\lambda_{\nu j}}{\lambda_{\nu k}}-1\right|^{2}
\end{aligned}
$$




$$
\begin{aligned}
& \geqq \sum_{\nu, j} \lambda_{\nu j} \sum_{k} \lambda_{\nu k}\left|\frac{\lambda_{\nu \jmath}}{\lambda_{\nu k}}-m_{\nu} \lambda_{\nu j}\right|^{2} \\
& =\sum_{\nu, j} \lambda_{\nu j} \sum_{k} \lambda_{\nu j} \frac{\lambda_{\nu j}}{\lambda_{\nu k}}\left|m_{\nu} \lambda_{\nu k}-1\right|^{2} \\
& \geqq \varepsilon^{2} \sum_{\nu, j} \lambda_{\nu j} \sum_{k} \frac{1}{m_{\nu}}\left|\left(m_{\nu} \lambda_{\nu k}\right)^{1 / 2}-1\right|^{2} \\
& =\varepsilon^{2} \sum_{\nu, k}\left|\left(\frac{1}{m_{\nu}}\right)^{1 / 2}-\lambda_{\nu k}{ }^{1 / 2}\right|^{2} .
\end{aligned}
$$

So, we obtain the convergence of the series standing last, which implies by the type $\mathrm{II}_{1}$ criterion that $(\boldsymbol{M})_{E}$ of Lemma 1 is a type $\mathrm{II}_{1}$ factor. (Or, it may happen that it is a finite type I factor.) Thus $M$ is a semi-finite factor, and is not of type III.

Our assertion being thus quite easily settled when (18) is satisfied, what we must do next is to reduce the general case to this case. To this end we will make repeated use of Lemma 2 .

The last paragraph of Section 2 states that the condition (17) is equivalent to the validity of

$$
\sum_{\nu, j, k} \lambda_{\nu_{j}} \lambda_{\nu_{k}}\left|\exp \left(2 \pi i t\left(\log \lambda_{\nu_{j}}-\log \lambda_{\nu_{k}}\right)\right)-1\right|^{2}<\infty
$$

for any real number $t$.

Put now

$$
\varphi_{\nu}{ }^{t}=\sum_{k} \lambda_{\nu k} \exp \left(-2 \pi i t \log \lambda_{\nu_{k}}\right) \text {. }
$$

Then, using again the fact that the mean square deviation becomes the least when one takes the mean value as the center, we see that

$$
\begin{aligned}
& \sum_{\nu, j, k} \lambda_{\nu j} \lambda_{\nu k}\left|\exp \left(2 \pi i t\left(\log \lambda_{\nu j}-\log \lambda_{\nu k}\right)\right)-1\right|^{2} \\
= & \sum_{\nu, j} \lambda_{\nu j} \sum_{k} \lambda_{\nu k}\left|\exp \left(-2 \pi i t \log \lambda_{\nu_{k}}\right)-\exp \left(-2 \pi i t \log \lambda_{\nu j}\right)\right|^{2} \\
\geqq & \sum_{\nu, j} \lambda_{\nu j} \sum_{k} \lambda_{\nu k}\left|\exp \left(-2 \pi i t \log \lambda_{\nu k}\right)-\varphi_{\nu}{ }^{t}\right|^{2} \\
= & \sum_{\nu, j} \lambda_{\nu j}\left|\exp \left(-2 \pi i t \log \lambda_{\nu j}\right)-{\varphi_{\nu}}^{t}\right|^{2} .
\end{aligned}
$$

By virtue of (20), we have

$$
\sum_{\nu, j} \lambda_{\nu_{j}}\left|\exp \left(-2 \pi i t \log \lambda_{\nu_{j}}\right)-\varphi_{\nu}{ }^{t}\right|^{2}<\infty
$$

and 
$(22)$

$$
\begin{aligned}
& \sum_{\nu}\left(1-\left|\varphi_{\nu}{ }^{t}\right|\right)^{2} \\
= & \left.\sum_{\nu, j} \lambda_{\nu j}|1-| \varphi_{\nu}{ }^{t}\right|^{2} \\
\leqq & \sum_{\nu, j} \lambda_{\nu j}\left|\exp \left(-2 \pi i t \log \lambda_{\nu j}\right)-\varphi_{\nu}{ }^{t}\right|^{2}<\infty .
\end{aligned}
$$

Define $\beta_{\nu}{ }^{t}$ as a real number satisfying

$$
\mathcal{P}_{\nu}{ }^{t}=\left|{\varphi_{\nu}}^{t}\right| \exp \left(-2 \pi i t \beta_{\nu}{ }^{t}\right) \text {. }
$$

Then (21) and (22) together imply

$$
\begin{aligned}
& \sum_{\nu, j} \lambda_{\nu j}\left|\exp \left(2 \pi i t\left(\beta_{\nu}{ }^{t}-\log \lambda_{\nu j}\right)\right)-1\right|^{2} \\
= & \sum_{\nu, j} \lambda_{\nu j}\left|\exp \left(-2 \pi i t \log \lambda_{\nu j}\right)-\exp \left(-2 \pi i t \beta_{\nu}{ }^{t}\right)\right|^{2} \\
\leqq & 2 \sum_{\nu, j} \lambda_{\nu_{j}}\left|\exp \left(-2 \pi i t \log \lambda_{\nu j}\right)-\varphi_{\nu}{ }^{t}\right|^{2}+2 \sum_{\nu, j} \lambda_{\nu_{j}}|1-| \varphi_{\nu}{ }^{t}||^{2}<\infty .
\end{aligned}
$$

Fix now a value of $t$. Later we will take $t=1 / 3$, but for a while, we don't specify $t$. For this value of $t$, we consider the set $\mathrm{I}^{\prime}(\nu)$ of $j$ satisfying

$$
\left|\exp \left(2 \pi i t\left(\beta_{\nu}{ }^{t}-\log \lambda_{\nu j}\right)\right)-1\right| \geqq \frac{1}{2} .
$$

Then, by virtue of (23),

$$
\begin{aligned}
\sum_{\nu} \sum_{j \in \mathrm{I}^{\prime}(\nu)} \lambda_{\nu j} & \leqq 4 \sum_{\nu} \sum_{j \in \mathrm{I}^{\prime}(\nu)} \lambda_{\nu j}\left|\exp \left(2 \pi i t\left(\beta_{\nu}{ }^{t}-\log \lambda_{\nu j}\right)\right)-1\right|^{2} \\
& \leqq 4 \sum_{\nu, j} \lambda_{\nu_{j}}\left|\exp \left(2 \pi i t\left(\beta_{\nu}{ }^{t}-\log \lambda_{\nu j}\right)\right)-1\right|^{2}<\infty .
\end{aligned}
$$

According to Lemma 2, we may thus leave those $\lambda_{\nu j}$ 's with $j \in \mathrm{I}^{\prime}(\nu)$ out of consideration, and we henceforth assume that

$$
\begin{aligned}
& \left|\exp \left(2 \pi i t\left(\beta_{\nu}{ }^{t}-\log \lambda_{\nu j}\right)\right)-1\right|<\frac{1}{2} \\
& \qquad \text { for } \nu=1,2, \cdots, \text { and } j=1, \cdots, m_{\nu} .
\end{aligned}
$$

Under this assumption,

$$
\begin{aligned}
& \left|\exp \left(2 \pi i t\left(\log \lambda_{\nu_{j}}-\log \lambda_{\nu_{k}}\right)\right)-1\right| \\
= & \left|\exp \left(2 \pi i t\left(\beta_{\nu}{ }^{t}-\log \lambda_{\nu_{k}}\right)\right)-\exp \left(2 \pi i t\left(\beta_{\nu}{ }^{t}-\log \lambda_{\nu_{j}}\right)\right)\right| \\
\leqq & \left|\exp \left(2 \pi i t\left(\beta_{\nu}{ }^{t}-\log \lambda_{\nu_{k}}\right)\right)-1\right|+\left|\exp \left(2 \pi i t\left(\beta_{\nu}{ }^{t}-\log \lambda_{\nu_{j}}\right)\right)-1\right| \\
< & 1 .
\end{aligned}
$$


From this, we conclude that for a suitable integer $n_{j k}$

$$
\left|2 \pi t\left(\log \lambda_{\nu_{j}}-\log \lambda_{\nu_{k}}\right)-2 n_{j k} \pi\right|<\frac{\pi}{3} .
$$

We take $t=1 / 3$ in this consideration, and then

$$
\left|\left(\log \lambda_{\nu_{j}}-\log \lambda_{\nu_{k}}\right)-3 n_{j k}\right|<\frac{1}{2} .
$$

So the $\lambda_{\nu j}$ 's $(j \in \mathrm{I}(\nu))$ are classed into several groups $\left\{\lambda_{\nu j} ; j \in \mathrm{I}(\nu, p)\right\}$ $\left(p=1, \cdots, l_{v}\right)$ such that

(24) $\quad\left|\log \lambda_{\nu_{j}}-\log \lambda_{v_{k}}\right|<1 \quad$ within a group,

(25) $\quad\left|\log \lambda_{\nu_{j}}-\log \lambda_{\nu_{k}}\right|>1 \quad$ between different groups.

We may consider that $\mathrm{I}(\nu, 1), \cdots, \mathrm{I}\left(\nu, l_{v}\right)$ are numbered so that

(26) $j \in \mathrm{I}(\nu, p), k \in \mathrm{I}(\nu, q)$ and $p<q$, then $j<k$.

Now we show that, except for a finite number of $\nu$ 's, we can find a $p=p_{v}\left(=1, \cdots, l_{v}\right)$ such that

$$
\sum_{j \in I\left(v, p_{\nu}\right)} \lambda_{\nu_{j}} \geqq \frac{1}{3} \text {. }
$$

Indeed, let $\Lambda$ be the set of $\nu$ for which one cannot find such a $p_{\nu}$. For $\nu \in \Lambda$, we are then able to take a $q_{\nu}\left(=1, \cdots, l_{\nu}\right)$ so that

$$
\sum_{p=1}^{q_{v}} \sum_{j \in \Gamma(v, p)} \lambda_{\nu_{j}} \geqq \frac{1}{3}, \sum_{p=q_{\nu}+1}^{l_{\nu}} \sum_{j \in[(\nu, p)} \lambda_{\nu_{j}} \geqq \frac{1}{3} .
$$

Writing $\bigcup_{p=1}^{q_{\nu}} \mathrm{I}(\nu, p)=\mathrm{I}_{1}(\nu), \bigcup_{p=q_{\nu}+1}^{l_{\nu}} \mathrm{I}(\nu, p)=\mathrm{I}_{2}(\nu)$, we have by (2), (25), and (26) that $\lambda_{\nu j} / \lambda_{\nu k}>2$ for $j \in \mathrm{I}_{1}(\nu), k \in \mathrm{I}_{2}(\nu)$, and, using (17),

$$
\begin{aligned}
\infty & >\sum_{\nu, j, k} \lambda_{\nu_{j}} \lambda_{\nu k} \min \left\{\left|\frac{\lambda_{\nu j}}{\lambda_{\nu k}}-1\right|^{2}, 1\right\} \\
& \geqq \sum_{\nu \in \Lambda} \sum_{j \in I_{1}(\nu)} \sum_{k \in I_{2}(\nu)} \lambda_{\nu j} \lambda_{\nu k} \min \left\{\left|\frac{\lambda_{\nu j}}{\lambda_{\nu k}}-1\right|^{2}, 1\right\} \\
& =\sum_{\nu \in \Lambda}\left(\sum_{j \in 1_{1}(\nu)} \lambda_{\nu j}\right)\left(\sum_{k \in I_{2}(\nu)} \lambda_{\nu k}\right) \\
& \geqq \sum_{\nu \in \Lambda} \frac{1}{3^{2}} .
\end{aligned}
$$

Therefore, $\Lambda$ is a finite set. 
Now we many leave the $\nu$ 's in $\Lambda$ out of consideration. And we assume that $(27)$ is valid for all $\nu(=1,2, \cdots)$. We claim then

$$
\sum_{\nu}\left(1-\sum_{j \in I\left(\nu, p_{\nu}\right)} \lambda_{\nu j}\right)=\sum_{\nu} \sum_{j \notin I\left(\nu, p_{\nu}\right)} \lambda_{\nu j}<\infty .
$$

Indeed, according to (17), (25), (26), (27),

$$
\begin{aligned}
& \infty>\sum_{\nu, j, k} \lambda_{\nu j} \lambda_{\nu k} \min \left\{\left|\frac{\lambda_{\nu j}}{\lambda_{\nu k}}-1\right|^{2}, 1\right\} \\
& \geqq \sum_{\nu}\left(\sum_{p<p_{\nu}} \sum_{j \in I(v, p)} \sum_{k \in \mathbb{I}\left(\nu, p_{\nu}\right)} \lambda_{\nu j} \lambda_{\nu k} \min \left\{\left|\frac{\lambda_{\nu j}}{\lambda_{\nu k}}-1\right|^{2}, 1\right\}\right. \\
& \left.+\sum_{j \in \mathrm{I}\left(v, p_{\nu}\right)} \sum_{p>p_{\nu}} \sum_{k \in \mathbb{I}(v, p)} \lambda_{\nu_{j}} \lambda_{\nu k} \min \left\{\left|\frac{\lambda_{\nu j}}{\lambda_{\nu k}}-1\right|^{2}, 1\right\}\right) \\
& =\sum_{\nu}\left(\sum_{p<p_{\nu}} \sum_{j \in \mathrm{I}(\nu, p)} \sum_{k \in \mathrm{I}\left(\nu, p_{\nu}\right)}+\sum_{j \in \mathrm{I}\left(\nu, p_{\nu}\right)} \sum_{p>p_{\nu}} \sum_{k \in \mathrm{I}(\nu, p)} \lambda_{\nu_{j}} \lambda_{\nu_{k}}\right) \\
& =\sum_{\nu}\left(\sum_{j \notin I\left(\nu, p_{\nu}\right)} \lambda_{\nu j}\right)\left(\sum_{k \in I\left(\nu, p_{v}\right)} \lambda_{\nu_{k}}\right) \\
& \geqq \frac{1}{3} \sum_{\nu} \sum_{j \notin \mathrm{I}\left(\nu, p_{\nu}\right)} \lambda_{\nu j}
\end{aligned}
$$

Now that the validity of (28) is thus established, we may let those $\lambda_{\nu j}$ 's with $j \notin \mathrm{I}\left(\nu, p_{v}\right)$ out of consideration by Lemma 2. But among $\lambda_{\nu j}$ 's with $j \in \mathrm{I}\left(\nu, p_{\nu}\right)$, we have by (24) that

$$
\left|\log \lambda_{\nu_{j}}-\log \lambda_{\nu k}\right|<1 \text {, }
$$

or $\lambda_{\nu_{j}} / \lambda_{\nu_{k}}$ is bounded below by a positive constant irrespective of $\nu$.

We thus have reduced the general case to the case where (17) holds, and the proof is complete.

4. We have shown that the condition (17) implies the semifiniteness of $\boldsymbol{M}$. But actually we had more. Indeed, an integral and precise examination of our proof reveals that a type II factor constructed as an infinite tensor product of type I factors is necessarily a hyperfinite $\mathrm{II}_{1}$ factor or a tensor product of a hyperfinite $\mathrm{II}_{1}$ factor with a type $\mathrm{I}_{\infty}$ factor. (This remark is due to $\mathrm{H}$. Araki.)

5. Finally, we add a few words about the relation with Moore's type classification of measures.

Moore's classification of the measure $\mu$ considered in Section 2 
is perfectly in accord with that of the corresponding infinite tensor product when $n_{\nu}=m_{\nu}$ for every $\nu$. Thus the condition (17) assures the existence of a $\sigma$-finite measure $\rho$ on $\Omega$ which is invariant under the action of $G$, and is equivalent to $\mu$. To construct explicitly the measure $\rho$, we can apply Moore's notion of restricted direct product of measures.

To establish that (17) implies the semi-finiteness of $M$, we had first to leave a finite number of $\mathrm{H}_{\nu}(\nu \in \Lambda)$ out of consideration, and for each remaining index $\nu$ to limit the set $\mathrm{I}(\nu)$ to a smaller finite set $\mathrm{J}(\nu)$. As the measure $\rho_{\nu}$ on $\Omega_{v}$ for $\nu \in \Lambda$, we take a measure giving mass 1 to each point. We denote by $\Omega_{v}{ }^{\prime}$ an arbitrary one point subset of $\Omega_{\nu}$. As the measure $\rho_{\nu}$ for $\nu \notin \Lambda$, we take a measure such that $\rho_{\nu}\left(\left\{\omega_{\nu j}\right\}\right)=1 / m_{\nu}{ }^{\prime}$ for $j \in \mathrm{I}(\nu)$, where $m_{\nu}{ }^{\prime}$ denotes the number of elements in J $(\nu)$. Define $\Omega_{\nu}{ }^{\prime}$ to be the subset of $\Omega_{\nu}$ whose points are $\omega_{\nu j}(j \in \mathrm{J}(\nu))$. Then form the restricted direct product $\rho$ of these measures $\rho_{\nu}$ using the sets $\Omega_{\nu}{ }^{\prime}$. $\rho$ will be the measure sought.

\section{References}

[1] Araki, H., A lattice of von Neumann algebras associated with the quantum theory of a free Bose field, J. Math. and Phys. 4 (1963), 1343-1362.

[2] Bures, D. J. C., Certain factors constructed as infinite tensor products, Comp. Math. 15 (1963), 169-191.

[3] Moore, C. C., Invariant measures on product spaces, Proceedings of the Fifth Berkeley Symposium, Vol. II, Part 2 (1967), 477-459.

[4] von Neumann, J., On infinite direct products, Collected Works, Vol. 3, 323-399. 